Volume 8, No.1.4, 2019

International Journal of Advanced Trends in Computer Science and Engineering

Available Online at http://www.warse.org/IJATCSE/static/pdf/file/ijatcse5781.42019.pdf

https://doi.org/10.30534/ijatcse/2019/5781.42019

\title{
NS2 Based Performance Comparison Study between DSR and AODV Protocols
}

\author{
Ahmed Mohammed Fahad ${ }^{1}$, Sameer Alani ${ }^{2}$, Sarmad Nozad Mahmood ${ }^{3}$, Nadia Mohammed Fahad ${ }^{4}$ \\ ${ }^{1}$ Faculty of computer system and software engineering, University Malaysia Pahang, Malaysia, ahmedsipher2010@yahoo.com \\ ${ }^{2}$ Faculty of Information and Communication Technology, Universiti Teknikal Malaysia Melaka (UTeM) Malaysia, itsamhus@gmail.com \\ ${ }^{2,3}$ Computer Technical Engineering, Alkitab University, Iraq, sarmadnmahmood@ gmail.com \\ ${ }^{4}$ Faculty of information technology, University of Al-Anbar, Iraq, ndosh582@gmail.com
}

\begin{abstract}
Mobile AD-HOC Network (MANETs) are useful when no traditional communication infrastructure exists, and have applications in military and other fields; MANET is a set of nodes capable of communicating with each other irrespective of infrastructure or centralized management. The mobility of system nodes leads them to be arranged and designed automatically. In addition, wireless networks have received extensive attention in the fields of communication systems owing to their prospective applications in different fields such as military, industrial and private area networks.

Moreover, wireless networks are easy to install, inexpensive, reliable, and do not need a fixed infrastructure, unlike the wired networks. In the MANETs, routing protocols play an essential role to discover the ways between the source and the destination. Generally, an appointed routing protocol system aims to address the difficulties of the progressively evolving topologies. It is possible to divide MANET routing protocols into three kinds: proactive, receptive and hybrid. In this report, routing protocol topology such as queue size and a number of nodes was altered and the efficiency metrics of two reactive routing protocols such as Ad hoc On-Demand Distance Vector AODV and Dynamic Source Routing DSR were contrasted in terms of average end-to-end delay, average throughput and packet delivery ratio.
\end{abstract}

Key words: MANET, Routing Protocols, Networking, AdHoc, QoS.

\section{INTRODUCTION}

Wireless ad-hoc networks have many different uses in our everyday lives, such as traffic data that are collected and distributed by vehicle navigation systems, researchers in a conference sharing resources, the shared use of hardware and software on nodes available to different people, or the detection of fires using sensors in a building. Such networks also have used in military operations, to sense enemy activity, and in disaster areas where the communication infrastructure has been destroyed. The nodes in MANETs have restricted connection times and resources [1], and are mainly used to connect users in areas without any conventional communication infrastructure. MANETs have the ability to self-configure and self-manage, although their nodes cannot be used continuously because of their low computational capacity and limited battery power [2]. Thus, nodes are only connected during communication and frequently disconnect from the network because of topology changes, link breakages, and network partitions. Hop-by-hop methods are used to establish network routes, as the transmission range of the nodes is limited. Each node shares fixed content or data over the network and manages its content [3]. Wireless networks enable wireless nodes to exchange information, and like fixed networks Can be categorized as networks based on infrastructure and networks with less network [4]. In infrastructure-based networks, Nodes interact via a base station or a point of access linked to a specified network such as the Internet, as shown in Figure 1. Infrastructure networks are generally reliable, as there is less chance of the network topology changing. The existence of a base station facilitates effective routing and resource management, as the routing among nodes is determined in a centralized manner.

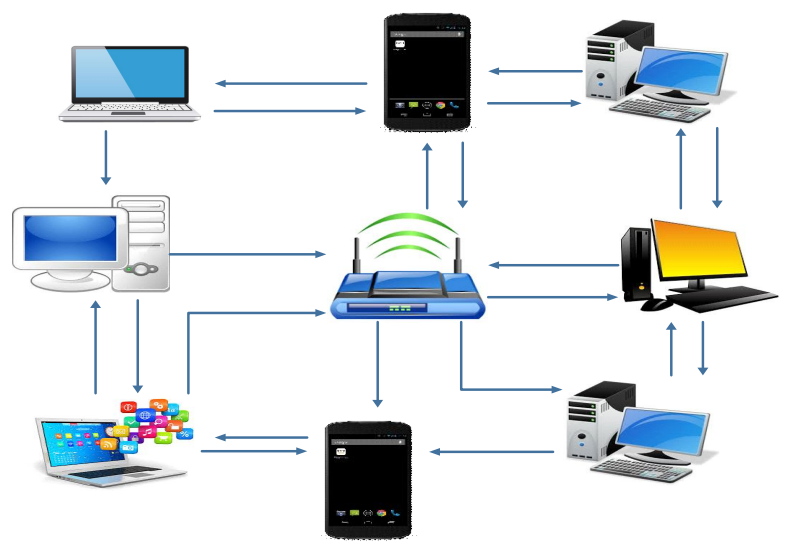

Figure 1: Communication between Wireless Nodes

Therefore, routing is defined as Process of information exchange in the network from one host to another [5]. Routing is also a mechanism for forwarding packets to the destination across the most effective way. The path efficiency is determined using various metrics such as the hop number, traffic, and security. Each host node works as a specific router in an ad-hoc network. Therefore, routing is an essential issue in MANETs [6], as finding and maintaining the primary obstacle to the design of an effective network protocol is the path from source to destination [7]. Many protocols have been proposed to deal with network limitations such as the high-security risk of mobile nodes and high inaccuracy rates [8]. The routing protocols can be categorized into the three main routing protocols shown in Figure 2. 


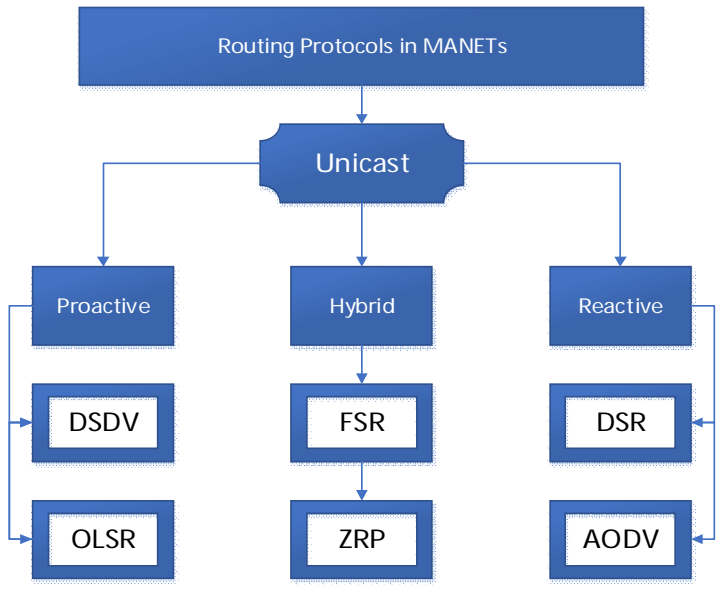

Figure 2: Routing Protocols Types

\section{CLASSIFICATION OF MANET}

MANET has three types of routing protocols as showing below:

\subsection{Proactive Routing Protocols}

Routing proactive or table-driven, routing protocols such as Destination-Sequenced Distance-Vector Routing (DSDV) maintain the current routing information, regardless of whether the paths are currently in use or not [9]. Each network node utilizes a routing table to transmit data packets and wants to connect to other network nodes. Proactive routing protocols require each node to maintain at least one table to store the routing information in order to maintain a coherent network perspective. If the network topology changes, the nodes, then distribute the latest information via the network. The routing table is broadcast and modified frequently to maintain stability. Such protocols have a unique way of communicating changes in the network structure and require a specific number of routing-related tables [7]. Proactive routing protocols have lower median delay times per packet and can access route information as and when it is needed. However, proactive protocols use a considerable amount of the network capacity to maintain the current and updated routing information, making them unsuitable for any reconfigurable MANETs.

\subsection{Reactive Routing Protocols}

Reactive, or on-demand, routing protocols do not retain routing information for nodes that are not communicating. In this way, routes that are not being used can be managed with lower overheads. However, Initial delays may happen when the reactive protocol searches for a path if one node decides to send a packet to another node, as a connection must be formed before the packet can be transmitted. The route discovery process in on-demand routing floods the network with RREQ packets [10].

There are two reactive protocol categories: routing source and routing hop-by-hop [11]. In source-routed protocols, the complete address from sender to receiver is carried by each data packet. Each intermediate node considers the data stored in the header part of each packet. Hence, to forward the packet to the destination, the intermediate nodes do not require updated routing data for each active route. In addition, there is no need to maintain neighbor connectivity by periodically broadcasting hello beacons. In the presence of fresh topology data, as each node has the ability to update its routing table, the paths can dynamically adapt to the evolving MANET setting. This transmits data packets to better and fresher routes[12 ].

\subsection{Hybrid Routing Protocols}

New-generation protocols are both reactive and proactive and are known as hybrid routing protocols [13]. The design of these new protocols allows nodes to work together when near. This reduces the route discovery overheads by forming a type of backbone, which increases scalability. Hybrid protocols proactively Keep paths for neighboring nodes, while also identifying routes to nodes that are far away with a route discovery technique. Therefore, each node sees the network as various zones or as being partitioned. Most hybrid protocols to date are regarded as being zone-based, although some group the nodes into clusters or trees [14]. Representative hybrid routing protocols area-based hierarchical link-state routing, scalable place update routing, Zone Routing Protocol (ZRP) and distributed tree-based routing [15].

\section{AD HOC ON DEMAND DISTANCE VECTOR (AODV)}

AODV Routing protocol through MANET in the detection of the AODV routing protocol process as described the source node telecast route request (RREQ) packet throughout MANET nodes [16]. In determines wait respond and time the RREQ includes information about routing such as the originator IP and broadcast ID and the sequence of target numbers. Each average node receives the RREQ packet and maintains the reverse path to the source and along with performing the first checks in whether received a Before using the same originator IP address and telecast ID, RREQ packet decides whether to ignore or accept RREQ packet. Secondly, if the RREQ packet in the event of the acceptance package average node-based verification number the stored sequence in its routing table [17].

\section{DYNAMIC SOURCE ROUTING (DSR)}

Dynamic Source Routing (DSR) facilitates the discovery of a route from the sender node to a receiver node across multiple network hops in an ad-hoc network [18]. In DSR, the packet headers contain a complete sequence of nodes to specify the forwarding route. As the packets include routing information, intermediate hops are not required to store the routing information, as illustrated in Figure 3.

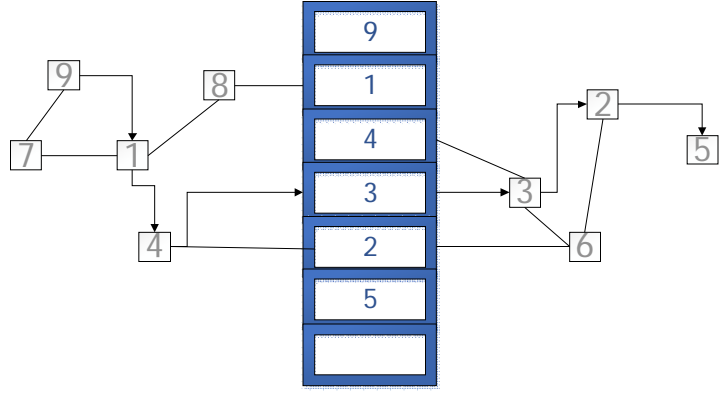

Figure 3: A Packet Being Routed from Node 9 to Node 5

As DSR does not involve router advertising or link status packets to be transmitted periodically, it has a lower overhead than other protocols. 
Furthermore, DSR can compute the correct routes for unidirectional links $[19,20]$. In the source routing, the sequence of nodes is determined by the node broadcasting the packet. The sequence is listed in the packet header using the node addresses. The DSR protocol incorporates routing, route discovery, and route maintenance. Route discovery refers to the mechanism employed by the source node to obtain a path to the destination. Similarly, the mechanism for detecting a break in the route and obtaining a corrected route is referred to as route maintenance.

\section{SIMULATION ENVIRONMENT}

In the simulation environment, the comparison between the two routing protocols has been done via NS-2.35 network simulator created to simulate a physically finished wireless ad-hoc multi-hop environment. Models of medium-level access control (MAC) and data link. The model is used is Random Waypoint Mobility due to its free movement. Two scenarios have done under this simulation. The first scenario is the number of nodes that varied from 10 up to 50 nodes., the second scenario is the queue size also varied from 10 to 50. All parameters are shown in Table 1.

Table 1: Simulation Parameters

\begin{tabular}{|c|c|c|}
\hline Parameter & Value & Unit \\
\hline Simulator & Ns-2 $(\mathrm{v} 2.35)$ & \\
\hline Simulation time & 150 & Sec \\
\hline Number of nodes & $10-20-30-40-50$ & Nodes \\
\hline Queue size & $10-20-30-40-50$ & \\
\hline Network area size & $600 \mathrm{~m} \times 500 \mathrm{~m}$ & $\mathrm{~m}^{2}$ \\
\hline Traffic type & CBR & \\
\hline
\end{tabular}

\section{PERFORMANCE METRIC}

There are various performance metrics used to assess the protocol. These metrics used to calculate the quantity of information acquired by destination, the number of packets drops; they require time to send information and the power consumption for the nodes in the network. In this paper, three performance metrics were used under two scenarios with several node and queue size as shown below:

\subsection{Packet Delivery Ratio (PDR)}

The proportion of information packets is acquired by destinations over the proportion of information packets sent by the source. It determines the rate of packet loss, which creates limits to the network; equation 1 shows how to collect the packet delivery ratio:

$$
\text { Average } P D R=\sum \frac{\text { Packet delivery ratio }}{\text { Packet Sent }}
$$

Figures (4 and 5) show the results of the two scenarios between the DSR and AODV protocols.

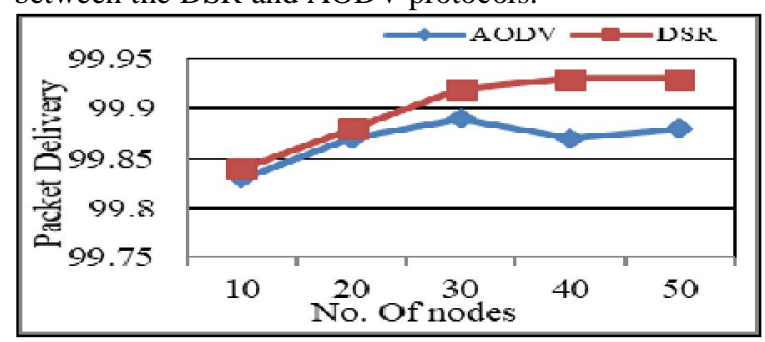

Figure 4: Packet Delivery Ratio with no. of Node

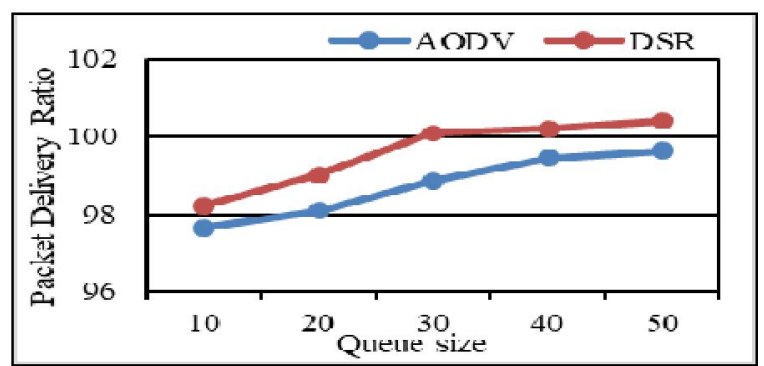

Figure 5: Packet Delivery Ratio with the Queue Size

\subsection{Throughput (TP)}

The number of bytes received successfully is called TP. Also, It is described as the real data packet that the target node receives. The most significant for best-effort traffic is the first two metrics. The routing load metric gives an evaluation of the routing protocol's efficiency in counting the throughput provided using equation 2 :

$$
\text { Throughput }=\sum \frac{\text { No of packet sent }}{\text { Time taken }}
$$

Figures (6 and 7) show the TP results in another scenario.

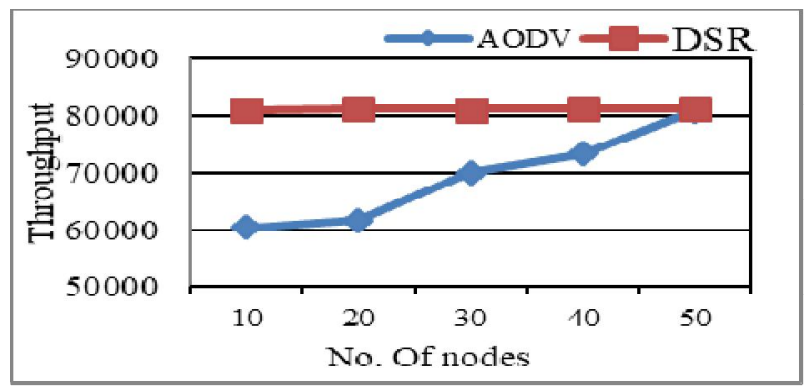

Figure 6: Throughput with no. of Node

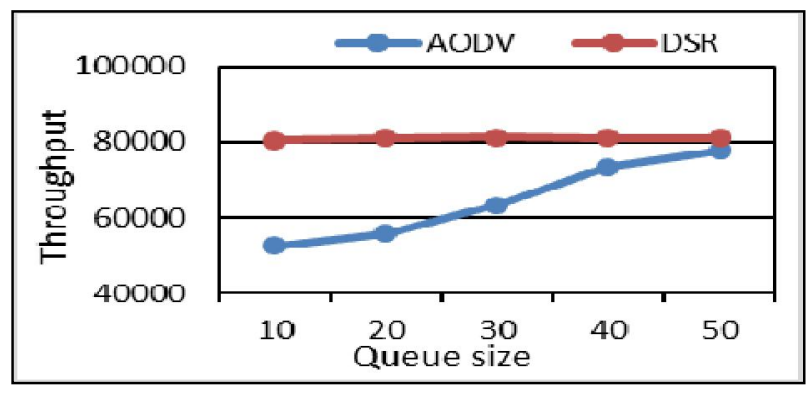

Figure 7: Throughput with the Queue Size

\section{A. Average End-to-End Delay (E2E delay)}

This term average E2E delay means the average time for data packets that are moving across the network from the source to the destination. This operation involves all potential delays, such as temporary storage during latency to discover the route Queuing interface and the transfer time. Equation 3 shows how to collect the E2E delay:

$$
\text { Avarage E2E delay }=\sum \frac{\text { Total E2E delay }}{\text { No.of packet sent }}
$$

The programming and the retransmission delay at the MAC as Shown in Figures (8 and 9). 


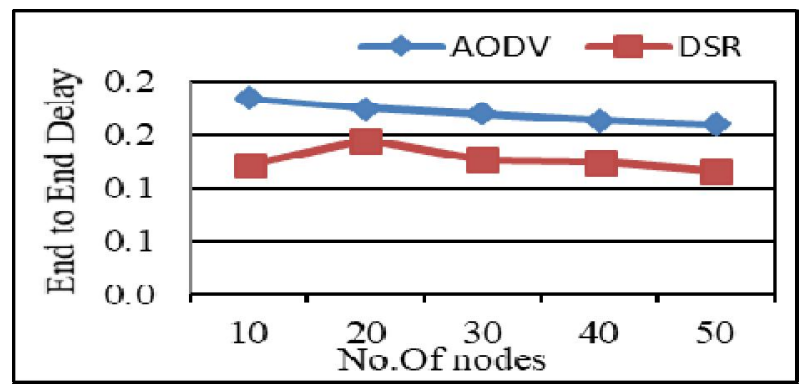

Figure 8: End to End Delay with no. of Node

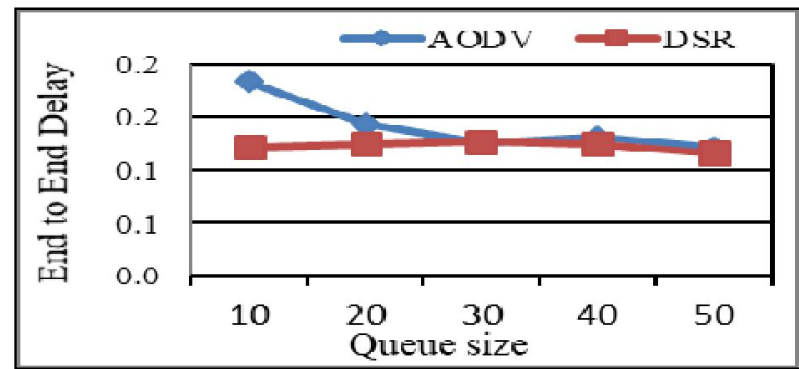

Figure 9: End to End Delay with the Queue Size

\section{RESULTS}

The results of two scenarios with tow protocols show that the first scenario number of node impacts on the performance of the protocol. This causes an increase in the nodes of the same area leading the protocol to choose the best path to send data. Besides, the data can reach the destination node by more than one path. Further, the queue size impact on the performance due to the traffic available in the queue leading the source node to send a new packet. It cannot be found a place in the queue so that the data will be dropped by way of increasing the queue size. This application enhances the performance of the routing protocols and can choose the DSR protocol to be better than AODV protocol in all performance matric due to the topology of the DSR routing protocol.

\section{CONCLUSION}

This paper presented the performance assessment of two kinds of AODV and DSR routing protocols. The comparison was performed using the random model mobility based on two parameters known as the number of nodes and the queue size. The set of nodes and the queue size have a significant effect on the routing protocols quality of services. Under the random waypoint elevated mobility model, the research of routing protocols has distinct behaviors, particularly when increasing the queue size and number of nodes. The efficiency of protocols changes the shipping percentage of packets in particular rises. The output of the AODV protocol can get variant by a number of node and queue size effects, particularly on E2E delay and throughput. however, the DSR protocol maintains its efficiency.

\section{REFERENCES}

[1] Abbas, N. I., Ilkan, M., \& Ozen, E. (2015). Fuzzy approach to improving route stability of the AODV routing protocol. EURASIP Journal on Wireless Communications and Networking, 2015(1), 235.

https://doi.org/10.1186/s13638-015-0464-5
[2] Abdulleh, M. N., Yussof, S., \& Jassim, H. S. (2015). Comparative study of proactive, reactive and geographical MANET routing protocols. Communications and Network, $7(02), 125$. https://doi.org/10.4236/cn.2015.72012

[3] Aggarwal, A., Gandhi, S., \& Chaubey, N. (2014). Performance analysis of AODV, DSDV and DSR in MANETS. arXiv preprint arXiv: 1402.2217.

[4] Bokhari, D. M., Hamatta, H. S., \& Siddigui, S. T. (2012). A review of clustering algorithms as applied in MANETs. International Journal of Advanced Research in Computer Science and Software Engineering, 2(11).

[5] Conti, M., \& Giordano, S. (2014). Mobile ad hoc networking: milestones, challenges, and new research directions. IEEE Communications Magazine, 52(1), 85-96. https://doi.org/10.1109/MCOM.2014.6710069

[6] Duggi, M. R. (2008). Apparatus and method for collecting active route topology information in a mobile ad hoc network: Google Patents.

[7] Fahad, A. M., \& Alani, S. (2017). A Security Framework Method Based on Harmony Search Algorithm to Prevent Malicious Nodes Attacks in MANETs. Advanced Science Letters, 23(6), 5217-5221. https://doi.org/10.1166/asl.2017.7345

[8] Fahad, A. M., \& Muniyandi, R. C. (2016). Research Article Harmony Search Algorithm to Prevent Malicious Nodes in Mobile Ad Hoc Networks (MANETs). https://doi.org/10.3923/itj.2016.84.90

[9] Giordano, S. (2002). Mobile ad hoc networks. Handbook of wireless networks and mobile computing, 325-346. https://doi.org/10.1002/0471224561.ch15

[10] Hussein, S.A. and D.P. Dahnil, A New Hybrid Technique to Improve the Path Selection in Reducing Energy Consumption in Mobile AD-HOC Networks. International Journal of Applied Engineering Research, 2017. 12(3): p. 277-282

[11] Han, L., Zhao, D., \& Zhou, M. (2005). A network layer security mechanism based on collaborative intelligent agents in MANET. Paper presented at the Information Technology: Research and Education, 2005. ITRE 2005. 3rd International Conference on.

[12] Jain, A. K., Tokekar, V., \& Singh, U. (2016). Detection and Avoidance of Integrated Attacks on MANET using Trusted Hyperbolic AODV Routing Protocol. Journal of Mobile Computing, Communications \& Mobile Networks, 3(2), 21-34.

[13] Jain, S., \& Sahu, S. (2012). Geometric Routing protocol based on genetic algorithm for minimized delay in MANETs. IJCSIT, 3(3), 4122-4126.

[14] Jubair, M., \& Muniyandi, R. (2016). NS2 Simulator to Evaluate the Effective of Nodes Number and Simulation Time on the Reactive Routing Protocols in MANET. International Journal of Applied Engineering Research, 11(23), 1139411399.

[15] Kaur, H., Sahni, V., \& Bala, M. (2013). A survey of reactive, proactive and hybrid routing protocols in MANET: A review. network, 4(3), 498-500.

[16] Khandelwal, V., \& Goyal, D. (2013). BlackHole Attack and Detection Method for AODV Routing Protocol in MANETs. International Journal of Advanced Research in Computer Engineering \& Technology (IJARCET), 2(4), pp: 1555-1559.

[17] S. Alani, Z. Zakaria, and H. Lago, "A new energy consumption technique for mobile Ad-Hoc networks," Int. J. Electr. Comput. Eng., vol. 9, no. 5, pp. 4147-4153, 2019.

[18] Kumar, J. M. S. P. J., Kathirvel, A., Kirubakaran, N., Sivaraman, P., \& Subramaniam, M. (2015). A unified approach 
for detecting and eliminating selfish nodes in MANETs using TBUT. EURASIP Journal on Wireless Communications and Networking, 2015(1), 143.

https://doi.org/10.1186/s13638-015-0370-x

[19] Royer, E. M., \& Toh, C.-K. (1999). A review of current routing protocols for ad hoc mobile wireless networks. IEEE Personal Commun., 6(2), 46-55.

https://doi.org/10.1109/98.760423

[20] Wozniak, M., Krishnaswamy, D., Callegari, C., TAKAGI, H., Prasad, N. R., Que, X., . . . Sandhu, R. (2015). Advances in Computing, Communications and Informatics (ICACCI). 